\title{
Adaptivity and Personalization in Ubiquitous Learning Systems*
}

\author{
Sabine $\mathrm{Graf}^{1}$ and Kinshuk ${ }^{2}$ \\ ${ }^{1}$ National Central University, Graduate Institute of Learning and Instruction, \\ No. 300, Jhongda Rd., Jhongli City, Taoyuan 32001, Taiwan \\ sabine.graflieee.org \\ ${ }^{2}$ Athabasca University, School of Computing and Information Systems, \\ 1 University Drive, Athabasca, AB T9S 3A3, Canada \\ kinshuk@ieee.org
}

\begin{abstract}
Ubiquitous learning is an emerging research field, allowing students to learn anywhere and anytime. Adaptivity and personalization issues play an important role in ubiquitous learning systems, enabling those systems not only to allow learners to learn whenever and wherever they want, but also in a personalized and adaptive way, considering the situation, characteristics, and needs of learners. In this paper, an overview of adaptivity and personalization issues in ubiquitous learning systems is provided and current and future research directions are discussed.
\end{abstract}

Keywords: Adaptivity, personalization, ubiquitous learning systems.

\section{Introduction}

Ubiquitous learning environments overcome the restrictions of classroom or workplace-restricted learning and extend e-learning by bringing the concepts of anytime and anywhere to reality, aiming at providing people with better educational experience in their daily living environments. Use of devices such as mobile phones and personal digital assistants (PDA) allows new opportunities for learners by being intensely connected. Therefore, educational content can be accessed and interaction can take place whenever learners need it, in different areas of life, regardless of space and time.

While "ubiquitous learning" is a growing research area, aspects of adaptivity and personalization become more and more important. Incorporating adaptivity and personalization issues in ubiquitous learning systems (ULS) allows these systems to provide learners with an environment that is not only accessible anytime and anywhere, but also accommodating to the individual preferences and needs of learners. Being aware of and considering the current context of the learners as well as, for example, their different prior knowledge, interests, learning styles, learning goals, and

* The authors would like to thank the National Science Council of the Republic of China, Taiwan, for financially supporting this research under Contract No. NSC 96-2520-S-008 -007 MY2 and NSC 097-2811-S-008-001-. The authors wish to acknowledge the support of iCORE, Xerox and the research related gift funding provided to the Learning Communities Project by Mr. Allan Markin. 
so on, leads to a more effective, convenient, and successful learning experience in the ubiquitous learning environments.

This paper is written in the context of the workshop on "Adaptivity and Personalization in Ubiquitous Learning Systems", aiming at giving an overview in this area and highlighting recent research. Section 2 introduces ubiquitous learning and Section 3 discusses adaptivity and personalization aspects in the context of ULSs. In Section 4, we give a brief introduction of the workshop papers and Section 5 concludes the paper with a brief discussion on future outlook.

\section{Ubiquitous Learning}

In this section, we give an overview about ubiquitous learning, describing its origin and definition and subsequently discussing the characteristics and features of ULSs.

\subsection{Origin and Definition of Ubiquitous Learning}

Ubiquitous learning has its origins in ubiquitous computing. David Lay describes ubiquitous computing as "a vision of computing power 'invisibly' embedded in the world around us and accessed through intelligent interfaces" [1]. The term "ubiquitous computing" has been introduced by Mark Weiser [2], who argued that the most profound technologies are those that are invisible and used by people unconsciously to accomplish everyday tasks. In a ubiquitous computing environment, many small computers are embedded in daily life objects, enabling these objects to support and assist people in tasks about work, education, and daily life. Important features of ubiquitous computing environments are the wireless communication between objects as well as the sensors, which allow the objects to sense user information and environment information in the real world and provide users with personalized services [3].

A ubiquitous learning system (ULS) supports learners through embedded and invisible computers in everyday life [4]. Such environments allow students to learn at any time and any place, encouraging them to more experiential learning [1] such as learning by doing, interacting and sharing, and facilitate on-demand learning, handson or minds-on learning and authentic learning [5].

Two other terms, namely mobile learning and pervasive learning, are often used in similar context with ubiquitous learning. Ogata and Yano [4] describe the difference of these terms based on Lyytinen and Yoo's work [6] about ubiquitous computing, considering the level of embeddedness and mobility. In mobile learning, learners carry their mobile devices with them, which act as their learning environment. Thus, mobile learning has a high degree of mobility but a low degree of embeddedness, since, apart from the web server, no other computers than the students' own mobile devices need to be involved in the learning process. Pervasive learning, on the other hand, takes place in an environment where small computers are embedded and communicate mutually in order to provide context-based information. Therefore, pervasive learning has a high degree of embeddedness but a low degree of mobility since learning depends on the location where the computers are embedded. Ubiquitous learning combines mobile and pervasive learning and assumes that computers are embedded in everyday objects. In ubiquitous learning, students move with their 
mobile devices which support their learning process by communicating with the embedded objects and other devices in the surrounding environment.

There exist also broader definitions of the term "ubiquitous learning". While Hwang, Tsai, and Yang [7] agree on the abovementioned definition by Ogata and Yano, they describe that a broader definition of ubiquitous learning focuses on the aspect of learning at any time and any place. According to this broad definition, an environment which allows students to learn wherever they are and whenever they want, can be considered as a ubiquitous learning environment. Furthermore, Ogata and Yano point out that for a broad definition of ubiquitous learning, both mobile learning and pervasive learning, are in the category of ubiquitous learning [4].

However, in this paper, we refer to the narrow-sense definition of ubiquitous learning given by Ogato and Yano [4], combining mobile and pervasive learning.

\subsection{Characteristics and Features of Ubiquitous Learning Systems}

Chen et al. [8] described five characteristics of mobile learning environments, from which Ogata and Yano [5] derived the following characteristics for ubiquitous learning: permanency means that students' work and the learning processes are recorded; accessibility means that learners can request and access their documents and data anywhere; immediacy allows students getting any information immediately, independent of their location; interactivity allows learners to interact with experts, teachers, and peers; and situating of instructional activities means that learning can be embedded in daily life, in a natural and authentic form. Bomsdorf [9] proposed additionally adaptability as the sixth characteristic of ULSs, referring to the function that learners get the right information at the right place and in the right way.

Hwang, Tsai, and Yang [7] also discussed the characteristics of ULSs, focusing more on aspects of context-awareness and adaptation. According to them, a ULS is context-aware, meaning that it can sense the learners' situation and the situation of the learners' current real world environment. Furthermore, a ULS is able to offer adaptive support based on the students' learning behavior and contexts in the real and cyber world. Moreover, the system can actively provide personalized support in the right way, at the right place, and at the right time, based on the personal and environmental contexts in the real world, as well as the learner's profile and learning portfolio. Another characteristic is that ULSs enable seamless learning, allowing students to move around in the real world. Furthermore, a ULS is able to adapt the learning material according to the functions of the mobile device the student is using.

A ULS can support students in many ways, offering them an environment where they can learn through experience in the real world, supported and guided by the system, which is able to adapt and personalize its interaction and suggestions to the learner. ULSs can support individual and collaborative learning. For individual learning, the system is able to interact with students, providing them with an active and student-centered way of learning [7]. The system can provide navigation support and guide students, based on their current location, to places where they can learn through experience or conduct location-dependent learning activities. Furthermore, the system is able to present learning material and suggest learning activities to the learners in a context-aware way, showing adaptive content or activities at the right time and the right place in order to facilitate a more authentic learning experience. For 
collaborative learning, ULSs can support learners in finding peers, with whom they can meet virtually or in real world and build learning groups, or experts whom they can ask for help and advice. Furthermore, when emphasizing on collaborative learning, aspects of social networks and Web 2.0 technologies can play an important role to facilitate communication, interaction, and knowledge sharing among students.

As stated, for example, by Hwang, Tsai, and Yang [7], Bomsdorf [9], and Yang [10], adaptivity and personalization are important functions of ULSs. Yang [10], for example, stated that ubiquitous learning is characterized by providing intuitive ways for identifying right collaborators, right contents, and right services in the right place at the right time based on the learners' surrounding context. Personalization and adaptivity deal with what "right" means for each learner. Therefore, the functions of personalization and adaptivity interweave with the features of ULSs mentioned above, making the interaction between the system and students, navigation support, content presentation, and searching for peers or experts personalized and adaptive.

In the next section, we discuss general issues about adaptivity and personalization as well as show how adaptivity and personalization can be applied in ULSs.

\section{Adaptivity and Personalization}

Adaptivity and personalization in learning systems refers to the function enabling the system to fit the students' current situation, needs, and characteristics, taking into account, for example, their knowledge level, learning styles, cognitive abilities, current location, motivation, interests, preferred language, and so on. While adaptivity focuses on the aspect that learners' situation, needs, and characteristics are considered by the system automatically, personalization is a more general term and highlights the customization of the system, including also issues which can be adapted and specified by learners themselves such as the color of the interface, the preferred language, or other issues which make the environment more personal.

Different aspects have to be considered when aiming at providing students with adaptive and personalized courses, material, or activities. One dimension incorporates what kind of information about the learner is used for adaptation. Another dimension considers what can be adapted in the system based on the specific information. In the following sections, these two dimensions are discussed in more detail.

\subsection{Which Information Is Used for Adaptivity and Personalization?}

Many research works about adaptivity and personalization have been carried out in the context of hypermedia and web-based learning systems, focusing for example on the consideration of students' level of knowledge, learning styles, cognitive abilities, motivation, and so on. In ULSs, this research is very relevant with respect to content presentation. Furthermore, information about the students' and the environments' context is a crucial factor for providing adaptivity and personalization in ULSs.

The necessary information for providing adaptivity and personalization in ULSs can be retrieved from different sources. Hwang, Tsai, and Yang [7] pointed out 5 types of situation parameters, which represent different kinds of information. Through the sensors of the ULS, information about the students' context and the environmental 
context can be gathered. Information concerning the students' context includes, for example, their current location, the time of their arrival and issues such as heartbeat or blood pressure. The environmental context includes information about the environment of a sensor, such as location, temperature, and information about approaching objects/people. Furthermore, information can be gathered from the students' interaction with the system via the mobile device, including for example stored documents, given answers to questions, and certain settings the learner made in his/her user interface. Moreover, the system can access a database, where personal data of students and environmental data are stored. Personal data can include the student's learning styles, course schedule, prior knowledge, progress in the course and so on. Environmental data provide more detailed information about the environment, such as a schedule of arranged learning activities or notes for using the site.

\subsection{What Can Be Adapted and Personalized?}

Another dimension refers to what can be adapted and personalized in a system. As mentioned above, a ULS can support learners by interacting with them, guiding them to suitable places, presenting them with learning material and activities, and helping them to interact with peers and experts. In the following paragraphs, examples for adaptivity and/or personalization for each of these features are discussed.

With respect to interaction between the system and the learner, ULSs can be actively involved in the students' learning activities [7]. Through their contextawareness, ULSs can provide personalized hints at the right time or suggest suitable learning activities for a learner, depending on the current environment and the needs of the student. An example for adaptive and personalized interaction between the system and the learner is shown in the language learning system JAPELAS [11]. JAPELAS aims at teaching foreign students Japanese polite expressions. When a learner starts talking to another person, the system gives a suggestion about the level of polite expression based on hyponymy, social distance, and situation through receiving information from the other person's mobile device and from sensors of the own device. Another example is the language learning system TANGO [12], which detects objects around the learner, using RFID tags, and involves these objects in learning activities, for example, asking the student to close a window or move a can from one place to the other.

In order to help learners to navigate to locations where learning can take place, personalization and adaptivity aspects deal mostly with location-awareness and planning suitable learning activities. For example, a ULS can generate a navigation path according to students' prior knowledge in order to remedy students' lack of knowledge [13]. Hwang, Tsai, and Yang [7] describe a similar scenario where a ULS asks a student to go to a specific place to observe and identify a plant.

Regarding content presentation, different methods exist for providing students with adapted and personalized learning material. These methods determine how learning material is presented differently for learners with different characteristics and needs. Brusilovsky [14] classified these methods regarding to their aim into two groups, namely for adaptive presentation and adaptive navigation support, whereby navigation in this context means how students navigate through the learning material. Many research works have been conducted, especially in the domain of adaptive educational 
hypermedia and web-based systems, focusing on generating courses and material that fit the students' profile and portfolio. Furthermore, for ULSs, adaptation of content presentation for a particular mobile device is another important issue [7]. Examples for applying adaptivity and personalization aspects for content presentation in ULSs are proposed by Bomsdorf [9] and Graf et al. [15]

Another feature of ULSs includes interaction among learners as well as between learners and teachers/experts. Adaptivity and personalization can be used for improving asynchronous and synchronous communication in a ULS. Regarding asynchronous communication, components such as discussion forums, question and answer services, and knowledge sharing services can be made more personal and adaptive to the learners' situation, characteristics, and needs. For example, considering where a student asked a specific question or generated a document can provide additional data, which can be useful when peers search for information. Furthermore, ULSs can help students to communicate synchronously by assisting them in forming learning groups or showing them who might be able to answer their questions. For example, Martin et al. [16] presented a location-based application which gives information about people who are close to the learner. Furthermore, a ULS can provide suggestions for building learning groups based on the students' location as well as other students' characteristics, as proposed, for example, in [15].

\section{Topics of the Workshop}

In this section, we outline the papers presented at the International Workshop on Adaptivity and Personalization in Ubiquitous Learning Systems, aiming at introducing current research in the respective area. Each of these papers contributes towards the development and the effectiveness of adaptive and personalized ubiquitous learning environments, focusing on different aspects.

The paper by Thalmann is a review paper on the adaptation criteria used in adaptive hypermedia systems, showing what kind of information is applied for providing adaptivity and personalization. Furthermore, suggestions are provided for preparing learning material with respect to the identified adaptation criteria.

The paper by Glavinić and Granić deals with adaptivity and adaptability issues for user interfaces of learning systems, concerning adaptation of knowledge presentation and interaction style as well as focusing on adaptation to mobile devices.

The next two papers discuss development issues. The paper by Hussain, Lechner, Milchrahm, Shahzad, Slany, Umgeher, and Wolkerstorfer deals with the development process of applications for mobile devices. In this paper, the integration of extreme programming and user centered design in the development process is proposed and its benefits are demonstrated. Mödritscher and Wild proposed an approach for personalized e-learning based on web application mashups, focusing on how the learning process can be adapted by allowing learners integrating various web-based tools into one aggregated view and supporting them in building and sustaining learning communities.

The paper by Zakrzewska deals with improving the formation process of learning groups. Zakrzewska presented an approach for clustering students according to their learning styles and usability preferences. This technique can contribute to the formation of learning groups in ULSs by suggesting learners other peers who are in the same cluster and therefore can learn together from the same material. 
The next two papers deal with facilitating and supporting learners with little experience with technology. Ziefle investigated which instruction format is beneficial for navigation performance in mobile phones as well as whether one instructional format can be used in general or the instruction format should be tailored to specific information needs and user abilities. Morandell, Hochgatterer, Fagel, and Wassertheurer presented a study about the usage of personalized photo-realistic avatars for elderly people and people with mild cognitive impairment, aiming at improving the acceptance and user-friendliness of ambient/ubiquitous assisted living environments.

\section{Conclusions}

This paper aimed at providing an overview about adaptivity and personalization issues in ULSs, by presenting an introduction about ubiquitous learning and discussing the possibilities of adaptivity and personalization in ULSs. Subsequently, we introduced current research in this area, presented at the International Workshop on Adaptivity and Personalization in Ubiquitous Learning Systems.

Ubiquitous learning is an emerging and promising research field, which can benefit strongly by considering personalization and adaptivity aspects, making the support provided to the learners more convenient and effective. As can be seen from the proposed research works, many areas such as mobile learning, ambient assisted living, human-computer interaction, and adaptive hypermedia need to contribute in the development and effective usage of adaptive and personalized ULSs.

As a conclusion from the past and current research directions, one issue seems to be of special importance for future research. ULSs can detect and store a huge amount of information about learners and their environments. However, currently only few kinds of information are considered in a single ULS when providing adaptive and/or personalized support. A great potential lies in considering and combining the huge amount of information, which can be detected by ULSs, and using it for providing richer and more effective adaptivity and personalization for learners. This includes especially the combination of context information from ULSs, such as location or data about the environment, with information in the learners' profile, such as his/her interests, motivation, cognitive abilities, and learning styles.

\section{References}

1. Lay, D.: Ubiquitous computing. In: Emerging technologies for learning, vol. 2, pp. 64-79. British Educational Communications and Technology Agency (2007)

2. Weiser, M.: The Computer for the 21st Century, September 2001, pp. 94-104. Scientific American (2001)

3. Hwang, G.-J.: Criteria and Strategies of Ubiquitous Learning. In: Proceedings of the IEEE International Conference on Sensor Networks, Ubiquitous, and Trustworthy Computing (SUTC 2006), pp. 72-77. IEEE Computer Society, Los Alamitos (2006)

4. Ogata, H., Yano, Y.: Context-Aware Support for Computer-Supported Ubiquitous Learning. In: Proceedings of the International Workshop on Wireless and Mobile Technologies in Education, pp. 27-34 (2004) 
5. Ogata, H., Yano, Y.: How Ubiquitous Computing can Support Language Learning. In: Proceedings of the International Conference on Knowledge Economy and Development of Science and Technology, pp. 1-6 (2003)

6. Lyytinen, K., Yoo, Y.: Issues and challenges in ubiquitous computing. Communications of ACM 45(12), 63-65 (2002)

7. Hwang, G.-J., Tsai, C.-C., Yang, S.J.H.: Criteria, Strategies and Research Issues of Context-Aware Ubiquitous Learning. Educational Technology \& Society 11(2), 81-91 (2008)

8. Chen, Y.-S., Kao, T.-C., Sheu, J.-P., Chiang, C.-Y.: A Mobile Scaffolding-Aid-Based Bird-Watching Learning System. In: Proceedings of the IEEE International Workshop on Wireless and Mobile Technologies in Education (WMTE 2002), pp. 15-22. IEEE Computer Society Press, Los Alamitos (2002)

9. Bomsdorf, B.: Adaptation of Learning Spaces: Supporting Ubiquitous Learning in Higher Distance Education. In: Mobile Computing and Ambient Intelligence: The Challenge of Multimedia, Dagstuhl Seminar Proceedings 05181 (2005)

10. Yang, S.J.H.: Context Aware Ubiquitous Learning Environments for Peer-to-Peer Collaborative Learning. Educational Technology \& Society 9(1), 188-201 (2006)

11. Yin, C., Ogata, H., Yano, Y.: JAPELAS: Supporting Japanese Polite Expressions Learning Using PDA(s) Towards Ubiquitous Learning. The Journal of Information and Systems in Education 3(1), 33-39 (2004)

12. Ogata, H., Akamatsu, R., Mitsuhara, H., Yano, Y., Matsuura, K., Kanenishi, K., Miyoshi, Y., Morikawa, T.: TANGO: Supporting Vocabulary Learning with RFID tags. In: Electronic proceedings of International Workshop Series on RFID, Tokyo (2004)

13. Chang, A., Chang, M.: Creating an Adaptive Mobile Navigation Learning Path for Elementary School Students Remedy Education. In: Proceedings of the International Conference on Interactive Computer Aided Learning, Villach, Austria (2006)

14. Brusilovsky, P.: Adaptive hypermedia. User Modeling and User-Adapted Interaction 11, 87-110 (2001)

15. Graf, S., MacCallum, K., Liu, T.-C., Chang, M., Wen, D., Tan, Q., Dron, J., Lin, F., McGreal, R.: Kinshuk: An Infrastructure for Developing Pervasive Learning Environments. In: Proceedings of the IEEE International Workshop on Pervasive Learning (PerEL 2008), pp. 389-394. IEEE Press, Hong Kong (2008)

16. Martín, S., Sancristobal, E., Gil, R., Castro, M., Peire, J.: Mobility through Location-based Services at University. International Journal of Interactive Mobile Technologies (iJIM) 2(3), 34-40 (2008) 